\title{
Ojos que no ven... ¿Qué podemos hacer para incluir más a la fracción subterránea en estudios de vegetación?
}

\author{
Luis López-Mársico ${ }^{1, \bigotimes ;}$; Sofía Pestoni ${ }^{2 / 3} ;$ Georgina Conti ${ }^{2 / 3} ;$ Natalia Pérez- \\ Harguindeguy ${ }^{2,3}$; Guillermo Martínez Pastur ${ }^{4}$ Priscila Pinto; ${ }^{5}$ Agustín Sarquis; \\ M. Fernanda Reyes ${ }^{5,6}$; Pablo L. Peri ${ }^{7}$ \& Gervasio Piñeiro ${ }^{5,8}$ \\ ${ }^{1}$ Instituto de Ecología y Ciencias Ambientales, Facultad de Ciencias, Universidad de la República, Uruguay. ${ }^{2}$ Facultad \\ de Ciencias Exactas, Físicas y Naturales, Universidad Nacional de Córdoba, Argentina. ${ }^{3}$ Instituto Multidisciplinario de \\ Biología Vegetal (IMBIV-CONICET), Córdoba, Argentina. ${ }^{4}$ Laboratorio de Recursos Agroforestales, Centro Austral de \\ Investigaciones Científicas (CADIC-CONICET), Argentina. ${ }^{5}$ Instituto de Investigaciones Ecológicas y Fisiológicas Vinculadas \\ a la Agricultura (IFEVA-CONICET), Facultad de Agronomía, Universidad de Buenos Aires, Argentina. ${ }^{6}$ Laboratorio de \\ Rehabilitación y Restauración de Ecosistemas Áridos y Semiáridos (LARREA), Facultad de Ambiente y Salud, Universidad \\ Nacional del Comahue, Argentina. ${ }^{7}$ INTA EEA-Santa Cruz-Universidad Nacional de la Patagonia Austral (UNPA)- \\ CONICET. ${ }^{8}$ Departamento de Sistemas Ambientales, Facultad de Agronomía, Universidad de la República, Uruguay.
}

\begin{abstract}
RESUMEN. Conocer la estructura y el funcionamiento de la fracción subterránea de la vegetación es fundamental para comprender numerosos procesos que ocurren en distintos niveles de organización. Sin embargo, dicha fracción fue menos estudiada que su contraparte aérea, principalmente por el gran esfuerzo que demanda muestrearla a campo y procesarla en el laboratorio. En la XXVIII Reunión Argentina de Ecología (Mar del Plata, 2018) se realizaron dos simposios sobre la importancia de conocer las raíces en estudios ecológicos. De los simposios surgió la necesidad de 1) cuantificar los estudios que hayan evaluado las fracciones subterránea y aérea de la vegetación, y 2) determinar las metodologías empleadas y las variables de la fracción subterránea registradas en sistemas naturales (i.e., pastizales, estepas, bosques, arbustales y desierto) y antropizados (i.e., pasturas y cultivos) en seis provincias fitogeográficas de la Argentina y en dos regiones geomorfológicas de Uruguay. Se registraron 933 estudios publicados entre 1990 y 2019. El 57\% y el 23\% correspondieron a estudios exclusivos de la fracción aérea y de la subterránea, respectivamente, con un incremento exponencial en el tiempo de ambas fracciones. En la actualidad existe una tendencia a incorporar el compartimiento subterráneo en estudios ecológicos. Mediante un análisis sistemático se encontró que se emplearon seis métodos de muestreo (barreno, ruleros, planta entera, monolitos, rhizotron y estimación de la biomasa subterránea a partir de la biomasa aérea) para evaluar cuatro variables (biomasa subterránea, productividad primaria neta subterránea, algunos atributos radicales y tasa de descomposición radical). El método más empleado fue el del barreno y la variable más evaluada fue la biomasa subterránea. Proponemos fomentar la colaboración entre equipos de investigación y establecer comparaciones metodológicas para comprender los alcances de los resultados y obtener estimaciones más confiables sobre las consecuencias del cambio en el uso del suelo.
\end{abstract}

[Palabras clave: raíces, biomasa subterránea, productividad primaria neta subterránea, atributos radicales, descomposición, Argentina, Uruguay]

Aвstract. Out of sight... What can be done in order to further include the belowground compartment in vegetation studies? To know the structure and functioning of the belowground vegetation compartment is essential to understand numerous processes that occur at different organization levels. However, the belowground vegetation compartment has traditionally been less studied than the above layer due to the great effort required for field sampling and laboratory processing. In the XXVIII Reunión Argentina de Ecología, Mar del Plata 2018, two symposia about the importance of root knowledge in ecological studies were conducted. From this exchange arose the need to 1) quantify studies that include data of belowground and aboveground vegetation, and 2) determine the methodologies and the variables of the belowground compartment recorded in natural (grasslands, steppes, forests, shrubs, and desert) and human modified systems (pastures, crops) in six Argentinean phytogeographic provinces and in two Uruguayan geomorphological regions. There were 933 published studies from 1990 to 2019 . The $57 \%$ and 23\% corresponded to exclusive studies of the above and belowground compartments respectively, with an exponential increase in the time of both fractions. Currently, there is a tendency to incorporate the underground compartment in ecological studies. Through systematic analysis, it was found that six sampling methods were used (soil core, ingrowth cores, trench, monoliths, rhizotron and belowground biomass estimation from aboveground biomass) where four variables of the belowground vegetation compartment were recorded (belowground biomass, belowground net primary productivity, root traits, and roots decomposition rate). Obtaining soil volumes by soil core was the most used method, while belowground biomass was the most evaluated variable. We propose to encourage collaboration between research teams and establish methodological comparisons to understand the scope of the results and obtain better estimates about the consequences of land-use change.

[Keywords: roots, belowground biomass, below-ground net primary productivity, root traits, decomposition, Argentina, Uruguay]

Editor asociado: Alejandro Bisigato $\bar{\triangle}$ luislopez@fcien.edu.uy
Recibido: 28 de Julio de 2019

Aceptado: 2 de Febrero de 2020 


\section{INTRODUCCIÓN}

Conocer la estructura y el funcionamiento de la fracción subterránea de la vegetación es clave para comprender numerosos procesos (e.g., secuestro de C, fertilidad del suelo y conservación de los recursos hídricos, entre otros) que ocurren a distintos niveles de organización en ecosistemas naturales o antropizados. La importancia de la fracción subterránea en estos procesos puede ser igual o mayor que la fracción aérea. A su vez, la fracción subterránea de la vegetación, en particular la biomasa subterránea (BS), se vincula de manera estrecha con la microbiota del suelo. En términos generales, los procesos que tienen lugar en el suelo fueron tratados como parte de una "caja negra" y menos explorados que los que ocurren a nivel aéreo. Menos del 20\% de los estudios de ecología vegetal a nivel global cuantifican alguna variable de la fracción subterránea (Wilson 2014). La BS es importante en algunos ecosistemas naturales (pastizales templados y tropicales, tundras y desiertos) en los que la relación biomasa subterránea:biomasa aérea (BS:BA) puede ser $>1$. Sin embargo, en otros ecosistemas naturales (bosques tropicales y bosques boreales), cultivos o plantaciones forestales, la BS:BA puede ser $<1$ (Jackson et al. 1996; Mokany et al. 2006; Wilson 2014; Qi et al. 2019).

El cambio en el uso del suelo es un fenómeno global que afecta la cobertura y el funcionamiento de los ecosistemas. Para el caso de Sudamérica, Volante et al. (2015) mostraron un incremento del $43 \%$ en la superficie cultivada entre 2000 y 2010 en cinco países estudiados. Para entender las consecuencias ecosistémicas de estos procesos de cambio es indispensable conocer tanto el comportamiento de la fracción aérea de la vegetación como el de la fracción subterránea. En la Argentina y Uruguay se repite el patrón observado a nivel global y tanto los procesos que tienen lugar en el suelo como la BS están menos estudiados. El gran esfuerzo físico, el tiempo que requieren los muestreos a campo y el procesamiento de las muestras suelen ser mencionados como las principales causas de la menor disponibilidad de información sobre la fracción subterránea de la vegetación a nivel global (Lauenroth 2000).

En el marco de la XXVIII Reunión Argentina de Ecología organizada en Mar del Plata en 2018 se realizaron dos simposios sobre temáticas complementarias: "Dinámica
Espacial y Temporal de la Biomasa Subterránea en Sistemas Naturales y Cultivos del Cono Sur de Sudamérica" y "Mirando lo que no Vemos: Ecología Subterránea". De estos simposios surgió la necesidad de realizar: 1) una cuantificación de los estudios que hayan evaluado la fracción subterránea y la aérea de la vegetación en la Argentina y Uruguay a través de una búsqueda bibliográfica sistemática, y 2) una revisión subjetiva de las metodologías empleadas y de las variables de la fracción vegetal subterránea estudiadas en sistemas naturales (pastizales, estepas, bosques, arbustales, desierto) y antropizados (pasturas, cultivos) de Argentina y Uruguay. En este contexto, y sobre la base del análisis de ejemplos representativos de estudios realizados en seis provincias fitogeográficas de la Argentina (de acuerdo a Oyarzabal et al. 2018) y dos regiones geomorfológicas de Uruguay (de acuerdo a Panario 1988, se propone abordar las siguientes preguntas: ¿Qué métodos de muestreo se emplean comúnmente para estudiar la fracción subterránea? ¿En qué se basa la decisión sobre la metodología empleada? ¿Cuáles son las variables evaluadas? ¿Cuáles son las ventajas y las restricciones de los estudios en macetas, en comparación con los realizados a campo? y ¿Cuáles son las perspectivas futuras sobre el tema?

\section{Estudios de la fracción subterránea y aérea de la vegetación}

Para cuantificar la proporción de estudios de la fracción subterránea en relación a los de la fracción aérea de la vegetación realizados en distintos ecosistemas de la Argentina y de Uruguay se llevó a cabo una búsqueda de artículos científicos publicados desde 1990 hasta octubre de 2019 en Scopus (búsqueda realizada el 8/10/2019), incluyendo las siguientes palabras clave: 1) para la fracción subterránea: (uruguay* OR argentin*) AND (biomass OR producti*) AND (plant OR vegetat* OR pasture* OR crop* OR grass* OR forest*) AND (belowground OR below-ground OR radical OR root) AND NOT ("root mean square" OR "free radical"), y 2) para la fracción aérea: (uruguay* ${ }^{*} \mathrm{R}$ argentin*) AND (biomass OR producti*) AND (plant OR vegetat* OR pasture* OR crop* OR grass* OR forest*) AND (aboveground OR above-ground OR aerial OR forage OR shoot).

Se registraron 933 estudios que cumplen con los requisitos buscados, de los cuales 529 trabajos (57\%) evaluaron sólo aspectos de la 


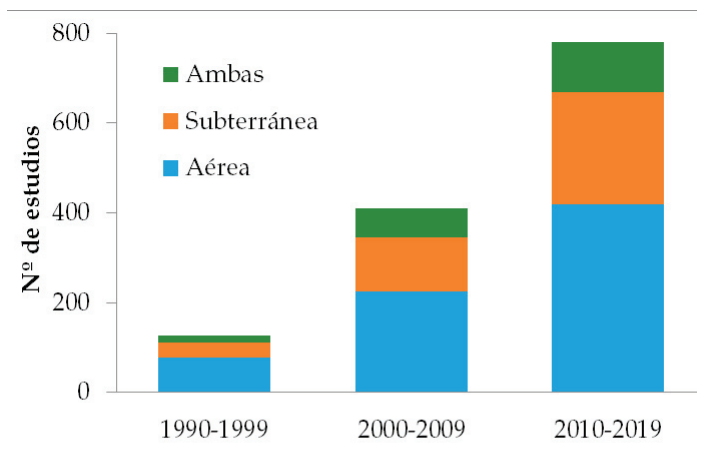

Figura 1. Número de estudios publicados exclusivamente sobre las fracciones aérea o subterránea de la vegetación y de los compartidos por ambas fracciones, desde 1990 hasta 2019 en la Argentina y en Uruguay.

Figure 1. Number of studies published exclusively on above or belowground vegetation compartments, and those shared by both compartments, from 1990 to 2019 in Argentina and Uruguay.

fracción aérea de la vegetación, 212 trabajos (23\%) realizaron mediciones solamente en la fracción subterránea y $192(20 \%)$ evaluaron ambas fracciones vegetales de manera conjunta. Estos resultados confirman que los estudios de la fracción subterránea son más escasos que los de la fracción aérea, aunque el número de estudios que involucran a la fracción subterránea duplica el $20 \%$ registrado por Wilson (2014). Al cuantificarlos por década, la cantidad de estudios aumenta exponencialmente con el tiempo $\left(\mathrm{y}=93.56 \mathrm{x}^{1.614}\right.$; $\mathrm{R}^{2}=0.999$ ) (Figura 1). Si bien la proporción de estudios que evalúan ambas fracciones no cambió en el tiempo, se observa un aumento no significativo en la proporción de estudios que evalúan algún aspecto de la porción subterránea $\left(\chi^{2}=1.033 ;\right.$ g.l. $\left.=2 ; P=0.60\right)$ (Figura 1$)$. Por lo tanto, actualmente existe una tendencia a incorporar más a la fracción subterránea en estudios ecológicos.

\section{Métodos de muestreo y decisión para su uso}

En los estudios recuperados en la búsqueda puede observarse que se aplicaron distintas metodologías para estimar variables estructurales, tales como la BS, la proporción de raíces vivas-muertas o finas-gruesas, y funcionales, como la productividad primaria neta subterránea (PPNS). Al igual que en otras decisiones metodológicas, los estudios reportan que la selección de métodos y diseños obedece al balance entre la eficiencia y los costos, la precisión y la robustez así como a la pregunta planteada y el sistema de estudio. Entre los métodos más empleados se puede mencionar el tradicional de calicatas, que consiste en realizar excavaciones para observar el sistema radical de la comunidad vegetal (Weaver 1915; Smit et al. 2000). Una modificación de este método, que se emplea sobre todo en sistemas en los que los individuos se encuentran dispersos en el espacio, es el muestreo denominado método de planta entera. Esta metodología prioriza la unidad de muestreo en profundidad del suelo. La separación del sistema radical se hace generalmente en el campo, por lo que es muy laborioso. El método de los monolitos, que consiste en obtener un cubo de suelo a una profundidad determinada y transportarlo al laboratorio para realizar el estudio (Böhm 1979), apunta a obtener mayor información de la distribución horizontal de la BS. El procesamiento en laboratorio permite separar, por técnicas complementarias, raíces vivas o muertas, finas o gruesas y otros órganos subterráneos. Además, si a estos cubos generados en el campo se les coloca un material transparente en sus paredes se lo denomina rhizotron y permite detectar in situ la dinámica temporal del sistema radical (Böhm 1979). Este método tiene la ventaja de estimar atributos radicales, como longitud, densidad o aparición-desaparición de raíces. Si en lugar de cubos se obtienen volúmenes cilíndricos de suelo, a través del empleo de barrenos, se puede estimar la BS fraccionada por profundidad de suelo, con un gran número de repeticiones (Bartos and Sims 1974; Smit et al. 2000). Por medio de mediciones sucesivas en el tiempo se puede estimar la PPNS (Lauenroth 2000). Usar el barreno puede ser útil para colocar tubos transparentes en los huecos generados y escanear el crecimiento radical por medio de una cámara que recorre internamente los tubos. Este método conocido como mini-rhizotron (Upchurch and Ritchie 1983) presenta la ventaja de obtener información continua a través de las imágenes, y las desventajas del costo del equipamiento y del efecto del tubo sobre la arquitectura de las raíces. Alternativamente, se pueden colocar mallas cilíndricas cribadas o ruleros en los huecos generados por el barreno, y rellenarlos con tierra sin raíces (Lund et al. 1970; Smit et al. 2000). Al cabo de un tiempo determinado se retiran los ruleros, por lo que este método permite determinar tasas de crecimiento de raíces en el tiempo; tiene la ventaja de ser menos costoso que el anterior. Estos tres últimos métodos pueden complementarse ya que el primero se puede emplear para obtener una estimación de la cantidad de BS y los siguientes permiten obtener atributos radicales y estimaciones de la PPNS. 
En los trabajos recopilados se identificaron algunos ejemplos de estudios, en su mayoría realizados por los autores de este artículo, en los que se detectaron distintos aspectos de la fracción subterránea de la vegetación y que representan la variedad de ecosistemas naturales y antropizados de la Argentina y de Uruguay. Se seleccionaron 35 estudios a partir de los cuales se construyó una base de datos que lista las metodologías empleadas, el tipo de vegetación considerado, las variables estimadas y la profundidad de suelo abordada (Tabla 1; Anexo 1). Algunos métodos de muestreo fueron usados en numerosas ocasiones (barreno, ruleros y planta entera), el de los monolitos en escasas oportunidades y otros dos en una única oportunidad (rhizotron y estimación a partir de la biomasa aérea) (Tabla 1; Figura 2). El método del barreno

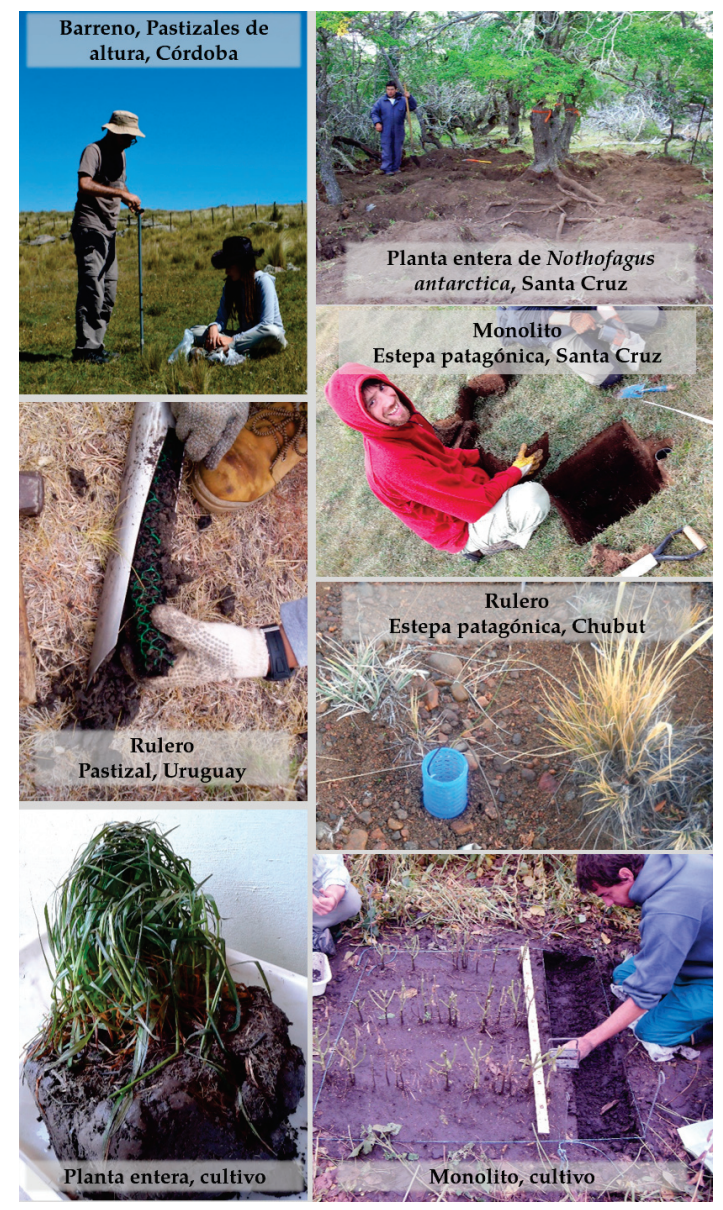

Figura 2. Métodos de muestreo empleados en distintas regiones de la Argentina y de Uruguay para obtener la fracción vegetal subterránea en sistemas naturales y en cultivos.

Figure 2. Sampling methods used in different regions of Argentina and Uruguay to obtain the vegetation belowground compartment in natural system and crops. fue el más utilizado, aplicado en casi todos los tipos de vegetación abordados, aunque mayormente en pastizales y estepas. Ha comprendido un rango de 0.2 a $1 \mathrm{~m}$ de profundidad del suelo y ha sido empleado en cuatro provincias fitogeográficas de la Argentina (en particular, en pastizales de las provincias Pampeana y Chaqueña) y en las dos regiones geomorfológicas de Uruguay (Tabla 1; Figura 2). El método de planta entera fue empleado en todos los tipos de vegetación, aunque en menor medida que el método anterior, y ha comprendido un rango de 0.05 a $3 \mathrm{~m}$ de profundidad del suelo. En su mayoría fue empleado en bosques y en estepas en las provincias Patagónica y Subantártica (Tabla 1; Figura 2). El método de los ruleros fue usado en igual proporción que el de planta entera, aunque sólo en pastizales, estepas y arbustales. Este método fue aplicado en un rango de 0.11 a $0.4 \mathrm{~m}$ de profundidad del suelo y fue empleado en tres provincias fitogeográfica de la Argentina y en una región geomorfológica de Uruguay (Tabla 1; Figura 2). El método de los monolitos ha sido utilizado en cuatro tipos de vegetación en tres provincias fitogeográficas de la Argentina y ha comprendido un rango de 0.1 a $1 \mathrm{~m}$ de profundidad del suelo (Tabla 1; Figura 2). Los métodos del rhizotron y el de la estimación de la BS desde la biomasa aérea han sido utilizados en bosques de las provincias fitogeográficas del Monte y Espinal, respectivamente (Tabla 1).

La elección del método de muestreo y el diseño experimental parecería estar basada en los métodos empleados previamente en el sistema de estudio de cada grupo de investigación. Es posible que esta decisión se relacione con la posibilidad de obtener resultados comparables, además de que el método esté avalado por trabajos científicos anteriores. Si bien se podría considerar que la asignación aleatoria de las parcelas de muestreo resulta más sencilla en sistemas de pastizales que en arbustales, estepas o bosques, la mayoría de las investigaciones ubican las parcelas de manera sistemática en todos los ecosistemas. La cercanía y el tamaño de los individuos parecería ser determinante tanto para muestrear cerca (e.g., bosques, arbustales, estepas) o lejos de ellos (e.g., pastizales, estepas). Por otra parte, aunque se conoce que el período del año de máxima productividad de raíces puede no coincidir con el de la productividad aérea, tal como ha sido registrado en la estepa patagónica (Soriano et al. 1987) y en pastizales (López-Mársico et al. 
Tabla 1. Métodos de muestreo y variables analizadas: biomasa subterránea (BS), productividad primaria neta subterránea (PPNS), atributos radicales (AR) y tasa de descomposición de raíces (Des) con sus respectivos rangos de profundidad del suelo, en 35 estudios en la Argentina y en Uruguay, abarcando distintos tipos de vegetación natural o antropogénica. Los valores entre paréntesis refieren al número de estudios en los que se obtuvo cada variable. $\mathrm{SD}=\sin$ dato, $\mathrm{NC}=$ no corresponde.

Table 1. Sampling methods and variables analyzed: belowground biomass (BS), belowground net primary productivity (PPNS), root attributes (AR), and roots decomposition rate (Des), with their respective soil depth ranges, in 35 studies of Argentina and Uruguay in different types of natural or human modified vegetation. Values in brackets refer to the number of studies in which each variable was obtained. $S \mathrm{D}=$ no data, $\mathrm{NC}=$ not applicable.

\begin{tabular}{|c|c|c|c|}
\hline Método & Vegetación & Variables & Profundidad (m) \\
\hline \multirow[t]{7}{*}{ Barreno } & Pastizal & $\begin{array}{l}\text { BS (7) } \\
\text { PPNS (5) }\end{array}$ & $0.2-1$ \\
\hline & Estepa & BS (5) & $0.2-0.3$ \\
\hline & Bosque & BS (1) & 0.6 \\
\hline & & $\mathrm{AR}(2)$ & \\
\hline & Arbustal & BS (1) & 0.3 \\
\hline & Pastura o Cultivo & BS (2) & 1 \\
\hline & & $\mathrm{AR}(1)$ & \\
\hline \multirow[t]{7}{*}{ Ruleros } & Estepa & BS (3) & 0.11 \\
\hline & & PPNS (1) & \\
\hline & & $\operatorname{Des}(1)$ & \\
\hline & Pastizal & BS (1) & 0.2 \\
\hline & & PPNS (2) & 0.25 \\
\hline & Arbustal & PPNS (2) & 0.4 \\
\hline & & $\mathrm{AR}(1)$ & \\
\hline \multirow[t]{7}{*}{ Planta entera } & Bosque & BS (4) & $0.5-3$ \\
\hline & Pastizal & BS (3) & 0.2 \\
\hline & & $\operatorname{Des}(1)$ & 0.05 \\
\hline & Estepa & $\mathrm{AR}(2)$ & $0.3-0.7$ \\
\hline & Arbustal & AR (2) & 3 \\
\hline & Desierto & BS (1) & 3 \\
\hline & Pastura o Cultivo & BS (1) & SD \\
\hline \multirow[t]{4}{*}{ Monolitos } & Pastizal & BS (2) & $0.4-1$ \\
\hline & Bosque & $\operatorname{Des}(1)$ & SD \\
\hline & Estepa & Des (1) & 0.1 \\
\hline & Pastura o Cultivo & BS (1) & SD \\
\hline Rhizotron & Bosque & $\mathrm{AR}(1)$ & 0.4 \\
\hline Estimación & Bosque & BS (1) & $\mathrm{NC}$ \\
\hline
\end{tabular}

2015), la mayoría de los muestreos de biomasa o productividad subterránea coinciden con el periodo de máxima biomasa o productividad aérea. Muchas veces, la decisión de emplear cierta metodología en un sistema dado basado en lo utilizado en estudios previos podría limitar la propuesta de nuevas metodologías. Por lo tanto, estas cuestiones metodológicas se deberían pensar de manera conjunta con la pregunta y el sistema de investigación, sin desconocer que el tipo de diseño seleccionado puede presentar riesgos que van desde grandes esfuerzos de muestreo hasta la dificultad para publicar los resultados.

\section{Principales variables evaluadas}

La BS fue la variable más evaluada con mayor frecuencia en la región (en 57\% de los estudios seleccionados), en particular utilizando el método del barreno (Tabla 1; Figura 2). La cantidad de BS puede considerarse como una "fotografía" en un momento dado. En función de la pregunta de investigación y del sistema de estudio se pueden discriminar por raíces finas o gruesas y otros órganos subterráneos o por raíces vivas o muertas, o pertenecientes a diferentes especies o grupos funcionales (e.g., hierbas, gramíneas, arbustos y árboles). La PPNS fue registrada en el 20\% de los estudios por medio de los métodos del barreno y ruleros (Tabla 1; Figura 2). Si bien se han planteado diferentes formas de estimar la PPNS (Dahlman and Kucera 1965), todas dependen de los datos de la cantidad de BS; puede considerarse como una "película" de la dinámica temporal de la cantidad de BS. Además, fueron evaluados distintos 
atributos radicales en el 16\% de los estudios, principalmente por medio de los métodos del barreno y planta entera (Tabla 1; Figura 2). Algunos de los atributos radicales evaluados fueron longitud, diámetro, densidad, fuerza de tensión y arquitectura radical. Por último, el $8 \%$ de los estudios evaluó la tasa de descomposición radical (Tabla 1). Si bien la estimación de la BS parece ser la principal variable de estudio, es necesario obtener otras variables complementarias que permitan determinar cómo las plantas responden a distintos factores ambientales en diferentes ecosistemas y cuál es la profundidad de enraizamiento o la de captación de agua por parte de las raíces (Bucci et al. 2009; Pereyra et al. 2017).

\section{El campo en una maceta: ventajas y restricciones}

La mayoría de los estudios en la región fueron realizados a campo, mientras que los estudios sobre la dinámica de la BS hechos en macetas fueron poco frecuentes. Los estudios en maceta pueden ser una herramienta de gran utilidad y complementaria a los estudios de campo para caracterizar los atributos subterráneos de la vegetación (e.g., longitud, densidad radical) y para entender ciertos aspectos de la dinámica del sistema radical a corto plazo (e.g., aparición-desaparición de raíces). Sin embargo, estos estudios no necesariamente repiten los patrones observados en el campo, ya que en las macetas las plantas suelen crecer en un medio sin recursos limitantes, al mismo tiempo que se restringe el volumen de suelo a ser explorado por el sistema radical. Todo esto puede interferir con los tratamientos aplicados y afectar los resultados del experimento (Poorter et al. 2012). Entonces, se debe tener en cuenta que los resultados obtenidos pueden sobre- o subestimar la cantidad de biomasa y las tasas de crecimiento subterráneas de las especies (Reyes and Aguiar, inédito). Al mismo tiempo, si bien estudios de este tipo son relativamente fáciles de lograr en especies herbáceas o plántulas de leñosas, no sucede lo mismo con adultos de arbustos o árboles, que requieren de una mayor infraestructura (e.g., contenedores adecuados) e inversiones de capital y tiempo de investigación.

\section{Perspectivas futuras}

Se registró un importante número de estudios enfocados en la fracción subterránea de la vegetación en una variedad de sistemas, aunque se observa una gran heterogeneidad de metodologías empleadas que atenta contra la comparación entre ecosistemas y regiones. Por otra parte, se detectó que algunos aspectos funcionales fueron poco abordados pese a su importancia en relación con los servicios ecosistémicos que se vinculan a ellos (e.g., secuestro de carbono, filtración de agua, fijación de suelo y protección de la erosión). Aunque no está explícito en los estudios, es evidente que los costos en términos económicos y en esfuerzo que conlleva el trabajo con la fracción subterránea de la vegetación son factores relevantes al momento de plantear las investigaciones. En este sentido, alrededor del mundo se generaron distintas redes de colaboración a escala regional entre equipos de investigación; estas redes facilitan el intercambio de metodologías y la construcción de bases de datos de mayor escala geográfica y temporal (e.g., DroughtNet, NutNet). Las colaboraciones en redes podrían permitir realizar, además, comparaciones metodológicas para comprender los alcances de los resultados obtenidos. A su vez, sería conveniente emplear metodologías complementarias para abordar una misma pregunta en sistemas similares y de esta manera evitar los sesgos propios de cada técnica. Si esto se lograra, se obtendrían mejores predicciones en relación a la interacción planta-suelo, y se arribaría a conclusiones más certeras sobre las consecuencias del cambio en el uso del suelo.

Agradecimientos. Agradecemos los comentarios y aportes de Sandra Bucci y de dos revisores anónimos ya que contribuyeron a lograr una mejor versión de este manuscrito.

\section{ReFERENCIAS}

Bartos, D., and P. Sims. 1974. Dynamics of a shortgrass Ecosystem. J Range Manage 27:33-36. https:/ /doi.org/10.2307/ 3896435.

Bucci, S. J., F. G. Scholz, G. Goldstein, F. C. Meinzer, and M. E. Arce. 2009. Soil water availability and rooting depth as determinants of hydraulic architecture of Patagonian woody species. Oecologia 160:631-641. https://doi.org/ 10.1007 /s00442-009-1331-z.

Böhm, W. 1979. Methods of studying root systems. Springer-Verlag, Berlin, Germany. https://doi.org/10.1007/9783-642-67282-8.

Dahlman, R., and C. Kucera. 1965. Root productivity and turnover in native prairie. Ecology 46:84-89. http:/ /doi.org/ $10.2307 / 1935260$. 
Jackson, R. B., J. Canadell, J. R. Ehleringer, H. A. Mooney, O. E. Sala, and E. D. Schulze. 1996. A global analysis of root distributions for terrestrial biomes. Oecologia 108:389-411. https://doi.org/10.1007/BF00333714.

Lauenroth, W. 2000. Methods of estimating belowground net primary production. Pp. 58-71 in O. E. Sala, R. B. Jackson, H. A. Mooney and R. W. Howart (eds.). Methods in Ecosystem Science. Springer-V. New York, USA. https: //doi.org/10.1007/978-1-4612-1224-9_5.

López-Mársico, L., A. Altesor, M. Oyarzabal, P. Baldassini, and J. Paruelo. 2015. Grazing increases below-ground biomass and net primary production in a temperate grassland. Plant Soil 392:155-162. https://doi.org/10.1007/ s11104-015-2452-2.

Lund, Z., R. Pearson, and G. A. Buchanan. 1970. An implanted soil mass technique to study herbicide effects on root growth. Weed Science 18:279-281. https://doi.org/10.1017/S0043174500079753.

Mokany, K., R. Raison, and A. Prokushkin. 2006. Critical analysis of root:shoot ratios in terrestrial biomes. Global Change Biol 12:84-96. https:/ / doi.org/10.1111/j.1365-2486.2005.001043.x.

Oyarzabal, M., J. Clavijo, L. Oakley, F. Biganzoli, P. Tognetti, I. Barberis, H. Maturo, R. Aragón, P. Campanello, D. Prado, M. Oesterheld, and R. J. León. 2018. Unidades de vegetación de la Argentina. Ecología Austral 28:40-63. https: //doi.org/10.25260/EA.18.28.1.0.399.

Panario, D. 1988. Geomorfología del Uruguay. Facultad de Humanidades y Ciencias, Universidad de la República, Montevideo, Uruguay.

Pereyra, D. A., S. J. Bucci, N. S. Arias, N. Ciano, P. M. Cristiano, G. Goldstein, and F. G. Scholz. 2017. Grazing increases evapotranspiration without the cost of lowering soil water storages in arid ecosystems. Ecohydrology 10:1-12. https: //doi.org/10.1002/eco.1850.

Poorter, H., J. Bühler, D. van Dusschoten, J. Climent, and J. Postma. 2012. Pot size matters: a meta-analysis of the effects of rooting volume on plant growth. Funct Plant Biol 39:839-850. https://doi.org/10.1071/FP12049.

Qi, Y., W. Wei, C. Chen, and L. Chen. 2019. Plant root-shoot biomass allocation over diverse biomes: A global synthesis. Global Ecol Conserv 18:e00606. https://doi.org/10.1016/j.gecco.2019.e00606.

Volante, J., J. Mosciaro, M. Morales Poclava, L. Vale, S. Castrillo, J. Sawchik, G. Tiscornia, M. Fuente, I. Maldonado, A. Vega, R. Trujillo, L. Cortéz, and J. Paruelo. 2015. Expansión agrícola en Argentina, Bolivia, Paraguay, Uruguay y Chile entre 2000-2010. Caracterización espacial mediante series temporales de índices de vegetación. Revista de investigaciones agropecuarias 41:179-191.

Smit, A., A. Bengough, C. Engels, M. van Noordwijk, S. Pellerin, and S. van de Geijn. 2000. Root methods: a handbook. Springer-Verlag, Berlin, Germany. https://doi.org/10.1007/978-3-662-04188-8.

Soriano, A., A. Golluscio, and E. Satorre. 1987. Spatial heterogeneity of the root system of grasses in the Patagonian arid Steppe. B Torrey Bot Club 114:103-108. https:/ / doi.org/10.2307/2996118.

Upchurch, D., and J. Ritchie. 1983. Root observation using video recording system in mini-rhizotrons. Agronomy Journal 75:1009-1015. https://doi.org/10.2134/agronj1983.00021962007500060033x.

Weaver, J. 1915. A study of the root-systems of prairie plants of Southeastern Washington. The Plant World 18:227248.

Wilson, S. 2014. Below-ground opportunities in vegetation science. J Veg Sci 25:1117-1125. https://doi.org/10.1111/ jvs.12168. 